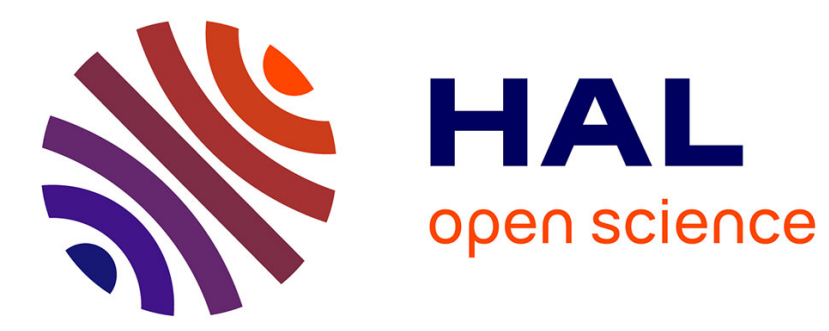

\title{
Pairwise Nash and Refereeing for Resource Allocation in Self-Organizing Networks
}

Mathew Goonewardena, Wessam Ajib, Elbiaze Halima

\section{To cite this version:}

Mathew Goonewardena, Wessam Ajib, Elbiaze Halima. Pairwise Nash and Refereeing for Resource Allocation in Self-Organizing Networks. 2014. hal-00995655

\section{HAL Id: hal-00995655 \\ https://hal.science/hal-00995655}

Preprint submitted on 23 May 2014

HAL is a multi-disciplinary open access archive for the deposit and dissemination of scientific research documents, whether they are published or not. The documents may come from teaching and research institutions in France or abroad, or from public or private research centers.
L'archive ouverte pluridisciplinaire HAL, est destinée au dépôt et à la diffusion de documents scientifiques de niveau recherche, publiés ou non, émanant des établissements d'enseignement et de recherche français ou étrangers, des laboratoires publics ou privés. 


\title{
Pairwise Nash and Refereeing for Resource Allocation in Self-Organizing Networks
}

\author{
Mathew Goonewardena *, Wessam Ajib ${ }^{\dagger}$, and Halima Elbiaze ${ }^{\dagger}$ \\ * École de Technologie Supérieure (ETS), Montréal, Québec, Canada, \\ *† Department of Computer Science, Université du Québec à Montréal (UQAM), Canada, \\ * mathew-pradeep.goonewardena.1@ens.etsmtl.ca, ${ }^{\dagger}\{$ ajib.wessam, elbiaze.halima $\} @$ uqam.ca
}

\begin{abstract}
This paper considers the problem of assigning frequency and time resources in a heterogeneous network, in a selforganizing manner. The general problem is to assign a resource set, so as to minimize the number of pairs of adjacent base stations that obtain the same resource. This can be modeled by Minimum-Collisions Coloring (MCC) on an undirected graph, where the colors are the resources, the vertices are the wireless nodes and the edges represent interference relations between nodes. The MCC decision problem is NP-complete. This paper develops a game-theoretic model for the MCC problem. The players of this game are a set of colored agents, which in practice could be software robots. The game is proven to possess multiple pure-strategy Nash Equilibria (NEs). Then a swapping mechanism is developed to improve the $\mathrm{NE}$ performance and the resulting coloring is pairwise Nash stable. Further refinement is proposed by making use of an external referee. All theoretical results are corroborated through simulations.
\end{abstract}

\section{INTRODUCTION}

Small Cells (SCs) are an economical and scalable solution to the ever increasing demand for throughput in wireless networks. The introduction of user deployed SCs, also called femtocells, presents network optimization problems, which were previously not addressed in classical operator deployed cellular networks. A wireless network with user deployed SCs is called a Heterogeneous and Small Cell Network (HetSNet) [1]. Just as any cell, SCs too require operating parameters to integrate into the wireless network. Complexity of SC configuration arises because of the large number and the unpredictability of their on-off operating times. These obstacles have given rise to an increased interest in Self-Organizing Networks (SONs) [2]. SONs address the large number and randomness of network elements by relegating the network intelligence, from a centralized system to the edge nodes themselves. Interactions among the nodes determine the state of the network.

One fundamental optimization problem in a HetSNet is the allocation of a finite set of resources, such that the pairs of adjacent Base Stations (BSs) that obtain a common resource are minimized. This is the Minimum-Collisions Coloring (MCC) problem, where the colors are the resources. Examples of MCC problems in HetSNets are, orthogonal frequency band distribution among interfering cells and scheduling time slots of a finite duration frame over a set of collocated interfering links. For the sake of generality, the rest of the paper refers to the finite resource set $\mathcal{N}$, as the set of colors.
MCC decision problem is NP-complete. Consider a reduction from proper-vertex coloring problem. Let $G=(\mathcal{V}, \mathcal{E})$ be an undirected graph, where $\mathcal{V}$ is the set of vertices and $\mathcal{E}$ is the set of undirected edges. The proper-vertex coloring decision problem is: can $G$ be $k$-colored where $k$ is a positive integer [3]? Call this problem $P_{1}(G, k)$. The MCC decision problem is; given the graph $G$ and two positive integers $N$ and $\tau$, is there an assignment of $N$ colors to the vertices such that the number of collisions is at most $\tau$ ? Call this problem $P_{2}(G, N, \tau)$. Clearly $P_{2}$ is in NP. $P_{1}(G, k)$ can be reduced, in polynomial time, to $P_{2}(G, k, 0)$. Since $P_{1}$ is NP-complete, it follows that $P_{2}$ is NP-hard. Since $P_{2}$ is NP-hard and is in NP, it is NP-complete. Hardness of the MCC problem permits us to seek heuristic efficient solutions.

Graph coloring heuristics have been extensively used in wireless networks [4]. This paper falls in the subclass of distributed game theoretic methods. Vertex coloring games have been the subject of study before. In [5] the proper-vertex coloring problem is modeled by a game played by the vertices. The actions are the set of colors. Through potential function and Best Response (BR) dynamics, the game is shown to converge to a Nash Equilibrium (NE) of proper coloring. In [6] the above work is extended to a distributed and parallel implementation. In [7] the bound for the worst case number of colors presented in [5] is improved. In [8] a proper coloring game is designed for the case when number of colors $k$ is at least $\Delta(G)+2$, where $\Delta(G)$ is the maximum degree of the graph. Any graph, $G$ can be proper $k$-colored in polynomial time and with knowledge of only the neighbors, if $k \geq \Delta(G)+1$. However the novelty of [8] is that the players follow a randomized strategy. In [9], the problem of assigning a fixed number of subchannels, $k$, among access points, is modeled by the graph coloring problem of finding weighted maximum induced $k$-colorable subgraph (weighted-Max-kCIS).

In the above discussed research work, the players of the games are the vertices, and most of the work concentrates on obtaining a proper coloring, while minimizing the number of colors used, except Max- $k$-CIS, which leaves some vertices uncolored. The research presented in this paper sets itself apart from previous work in three significant aspects. The first novelty is in the design of the game. The game of this paper, is played not by vertices, instead by a set of colored agents, and vertices are the strategy space of each colored agent. In a practical implementation the colored agents are akin to 


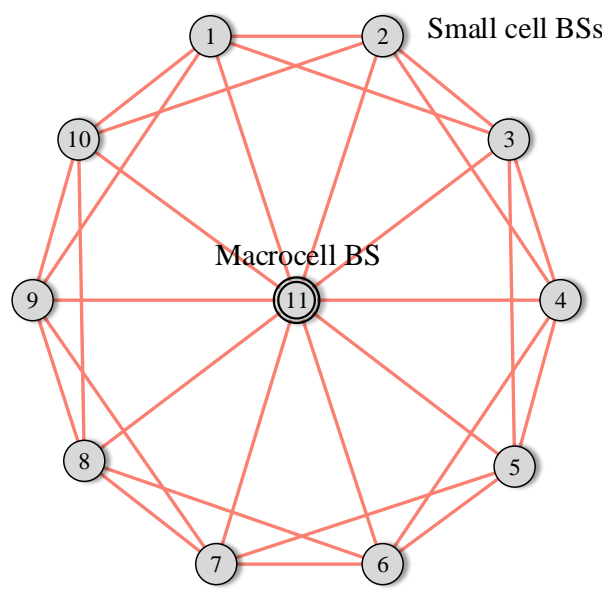

Graph, $G$
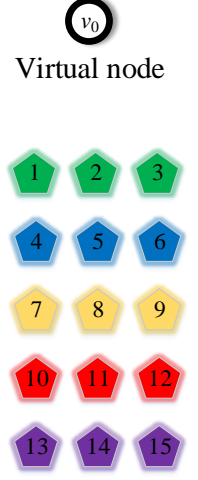

5 subchannels 3 agents per subchannel

Figure 1. The graph $G$ is designed to discourage common frequencies to nearby BSs. A set of 5 subchannels must be distributed. The operator creates 3 agents per subchannel.

software robots, currently used in Internet applications. Second significant difference is in the definition of the problem. In MCC the number of colors available is a predetermined parameter of the problem and therefore there exists no space to introduce extra colors as in proper coloring games. For that reason, the objective is to minimize the collisions, rather than to obtain a zero collision state. Also unlike Max- $k$-CIS, MCC does not leave any vertex uncolored. Finally, while all previous work considers only NE as the choice of equilibrium, this paper goes beyond, with pairwise Nash stability, which is a stronger criterion than NE. To the best of our knowledge this is the first paper to consider an MCC game among colored agents.

The remainder of the paper is organized as follows. Section II formulates the non-cooperative game. Section III improves NE performance by pairwise Nash stability. Section IV introduces a further refinement of pairwise Nash stability by involving an external refereeing process. Section $\mathrm{V}$ presents numerical results and finally Section VI concludes the paper.

\section{Non-COOPERATIVE GAME Formulation}

Let $G=(\mathcal{V}, \mathcal{E})$ be an undirected graph, where $\mathcal{V}$ is the set of vertices and $\mathcal{E}$ is the set of edges. Let $V$ be the cardinality of set $\mathcal{V}$, and the set of adjacent vertices of a vertex $v \in \mathcal{V}$ is denoted by $\mathcal{V}_{v}$. Two vertices are adjacent if they share an edge. The finite set of distinct colors is $\mathcal{N}:=\{1, \ldots, N\}$. A non-cooperative game in normal form is determined by a set of players, the actions/strategies available to the players, and the utility functions of them. The conceptual vision of the game that is developed in this paper is; given the graph $G$, we release a sufficiently large set of colored agents on to $G$. It is possible that some agents share the same color. Then each colored agent attempts to occupy a single vertex $v \in \mathcal{V}$ for itself. Thus the strategy space of each agent is the set of vertices. The cost that agent $\ell$ obtains by occupying vertex $v$ is an increasing function of the number of adjacent vertices to $v$, which are occupied by agents of the same color as $\ell$.

Let $\mathcal{L} \ni \ell$ denote the set of agents and $L$ its cardinality. In order to uniquely identify the colored agents, who share the same color, each agent is given a pair of values. The first element of $(x, y)$ identifies the color and the second element identifies the index, which is unique among agents of the same color. They are called indexed-colored agents or, in short, agents. Note that the exact indexing process is irrelevant, as long as each colored agent can be identified uniquely. For example if the graph $G$ has three vertices and two colors are available, $\mathcal{N}=\{1,2\}$, then the game requires that at least one color is duplicated. Therefore the resulting indexed-colored agents can be $\mathcal{L}_{1}=\{(1,1),(1,2),(2,1)\}$ or equivalently $\mathcal{L}_{2}=\{(1,1),(1,2),(2,3)\}$. One may prefer $\mathcal{L}_{2}$ indexing over $\mathcal{L}_{1}$, since the total number of agents can be directly read off the sorted second element in $\mathcal{L}_{2}$, which in this case is 3 . Clearly the minimum set of elements in $\mathcal{L}$ has to be equal to the number of vertices in the network, i.e., $L \geq V$. However there is no restriction on the upper limit of $L$, other than the demand to be finite. It is easy to realize that a set of indexed-colored agents with $V$ instances of each color $n \in \mathcal{N}$ is sufficient to obtain any combination of color assignments over $V$ vertices.

The NE of the game must ensure that two agents $\ell, \ell^{\prime} \in \mathcal{L}$ do not select the same vertex $v \in \mathcal{V}$ at an equilibrium state. Also at a NE, each vertex needs at least one agent. To address these two design requirements, this paper introduces two mechanisms. First, a virtual vertex $v_{0}$, which does not possess any neighbors, and second, a very large cost is associated to each agent occupying a vertex $v \in \mathcal{V}$, (but not $v_{0}$ ), if there are more than one agent in $v$. As is demonstrated later, the virtual agent and pricing scheme, together, ensure that the above mentioned two design requirements are met at the NE. Fig. 1 depicts a typical graph of a wireless network frequency assignment problem. The graph $G$ is such that each SC BS is adjacent, i.e., has an edge, to its closest and second closest neighbors and to the macrocell $\mathrm{BS}$. This means, the operator wants to discourage a SC from having a frequency band that is used by its close neighbors or the macrocell. Then fifteen agents are created, duplicating each subchannel 3 times.

Let $\mathcal{V}_{0}:=\mathcal{V} \cup\left\{v_{0}\right\}$ and let the number of agents occupying vertex $v \in \mathcal{V}$ be denoted by $\#(v)$. Let $S_{v} \subset \mathcal{N}$ denote the colors of the agents occupying vertex $v \in \mathcal{V}, t_{\ell} \in \mathcal{N}$ denote the color of agent $\ell \in \mathcal{L}$, and $q_{\ell} \in \mathcal{V}_{0}$ denote the vertex occupied by agent $\ell \in \mathcal{L}$. Note $t_{\ell} \in S_{q_{\ell}}$. Following standard game theory notation, $\mathbf{q}_{-\ell}:=\times_{\ell^{\prime} \neq \ell} q_{\ell^{\prime}}$ is the strategy vector of all players except $\ell$. Then for constants $a, d>0$, the linear cost function of an agent $\ell \in \mathcal{L}$ is given by,

$$
\begin{cases}a\left|\left\{v^{\prime}: t_{\ell} \in S_{v^{\prime}}, v^{\prime} \in \mathcal{V}_{q_{\ell}}\right\}\right|+d & \mathrm{c}_{\ell}\left(q_{\ell}, \mathbf{q}_{-\ell}\right):= \\ & \text { if } \#\left(q_{\ell}\right)=1 \\ (a+2) V+d & \text { and } q_{\ell} \in \mathcal{V}, \\ & \text { if } \#\left(q_{\ell}\right)>1 \\ (a+1) V+d & \text { and } q_{\ell} \in \mathcal{V}, \\ & \text { if } q_{\ell}=v_{0},\end{cases}
$$

and the total system cost, also called the social cost is,

$$
\mathrm{C}_{\mathcal{L}}\left(q_{\ell}, \mathbf{q}_{-\ell}\right):=\sum_{\ell \in \mathcal{L}} \mathrm{c}_{\ell}\left(q_{\ell}, \mathbf{q}_{-\ell}\right) \text {. }
$$


The finite normal form game thus performed by the coloredindexed agents is defined by $\mathcal{G}_{\mathcal{L}}:=\left\langle\mathcal{L}, \mathcal{V}_{0}^{L},\left\{\mathrm{c}_{\ell}(\cdot) \mid \ell \in \mathcal{L}\right\}\right\rangle$, where $\mathcal{V}_{0}^{L}:=\times_{\ell \in \mathcal{L}} \mathcal{V}_{0}$, is the joint action space of $L \geq V$ agents. From the above definition of the cost function (1) an agent $\ell$, always prefers an unoccupied vertex $v \in \mathcal{V}$ over $v_{0}$. Yet the agent prefers $v_{0}$ over occupying a vertex in $\mathcal{V}$ that is already occupied by another. And out of all vertices in the set $\mathcal{V}$, the agent prefers the vertex, which has the least number of collisions.

Next we explore the existence and properties of purestrategy NEs of $\mathcal{G}_{\mathcal{L}}$. As discussed before, it is paramount, to the applicability of game $\mathcal{G}_{\mathcal{L}}$, that no pure-strategy NE exists where a vertex in $\mathcal{V}$ has more than one agent, or has no agent. The following Proposition 1 proves just this, i.e., at any purestrategy $\mathrm{NE}$ of $\mathcal{G}_{\mathcal{L}}$, each vertex in $\mathcal{V}$ (note, not $v_{0}$ ) has exactly one agent. Conversely Proposition 2 proves that all one-to-one assignments between $\mathcal{V}$ and subsets of $\mathcal{L}$ of cardinality $V$ are pure-strategy NEs of $\mathcal{G}_{\mathcal{L}}$.

Proposition 1. There does not exist a $N E$ of $\mathcal{G}_{\mathcal{L}}$ where $\#(v)>1$ or $\#(v)=0, \forall v \in \mathcal{V}$.

Proof: Suppose agents $\ell, \ell^{\prime} \in \mathcal{L}$ are both occupying $v \in \mathcal{V}$. Then by definition of cost function in (1), any one of the agents can move to $v_{0}$ and obtain a strictly lower cost ,therefore the current strategy profile is not a best responses and therefore is not a NE. Now suppose there is a vertex $v \in \mathcal{V}$ with no color. By definition of the game, $L \geq V$. Therefore, there must be at least one agent $\ell \in \mathcal{L}$ with $q_{\ell}=v_{0}$ and this agent is strictly better off by occupying $v$. Therefore multiplecolored and uncolored vertices cannot be in any NE.

Proposition 2. A strategy vector, where each vertex in $\mathcal{V}$ obtains a single color, is a $N E$ of $\mathcal{G}_{\mathcal{L}}$.

Proof: Consider an allocation where each vertex in $\mathcal{V}$ has exactly one agent. Without loss of generality let $q_{\ell}=v$, $v \in \mathcal{V}$. If $\ell$ moves to another vertex in $\mathcal{V}$ its cost strictly increases. Similarly according to (1) if $\ell$ moves to $v_{0}$ its cost strictly increases. Therefore $v \in \mathcal{V}$ is a best response for agent $\ell$. Now consider an agent $\ell^{\prime}$ occupying $v_{0}$. Analogous to the above argument, moving to a vertex in $\mathcal{V}$ strictly increases the cost of $\ell^{\prime}$. Therefore $\ell^{\prime}$ is also playing a best response. Since each agent is at a best response, by definition the allocation is a NE. Therefore all allocations of agents where each vertex in $\mathcal{V}$ is occupied by one agent are pure strategy NEs of $\mathcal{G}_{\mathcal{L}}$.

It can also be shown that sequential BR dynamics converges to a $\mathrm{NE}$ of game $\mathcal{G}_{\mathcal{L}}$. Proposition 3 proves so by a potential function argument.

Proposition 3. The social cost $\mathrm{C}_{\mathcal{L}}(\cdot)$, is a generalized ordinal potential function of the game $\mathcal{G}_{\mathcal{L}}$.

Proof: For $\mathrm{C}_{\mathcal{L}}(\cdot)$ to be a generalized ordinal potential function the following condition must be satisfied:

$\mathrm{c}_{\ell}\left(q_{\ell}^{\prime}, \mathbf{q}_{-\ell}\right)-\mathrm{c}_{\ell}\left(q_{\ell}, \mathbf{q}_{-\ell}\right)<0 \Rightarrow \mathrm{C}_{\mathcal{L}}\left(q_{\ell}^{\prime}, \mathbf{q}_{-\ell}\right)-$ $\mathrm{C}_{\mathcal{L}}\left(q_{\ell}, \mathbf{q}_{-\ell}\right)<0$. That is, if $\ell \in \mathcal{L}$ reduces its cost by changing its strategy from $q_{\ell}$ to $q_{\ell}^{\prime}$ then the social cost also reduces. This can be proven by considering the two cases where a reduction in cost is possible. Case 1: both $q_{\ell}, q_{\ell}^{\prime} \in \mathcal{V}$ and $q_{\ell}^{\prime}$ is unoccupied before the change and, $\left|\left\{v^{\prime}: t_{\ell} \in S_{v^{\prime}}, v^{\prime} \in \mathcal{V}_{q_{\ell}^{\prime}}\right\}\right|<\left|\left\{v^{\prime}: t_{\ell} \in S_{v^{\prime}}, v^{\prime} \in \mathcal{V}_{q_{\ell}}\right\}\right|$. Case 2: $q_{\ell}=v_{0}, q_{\ell}^{\prime} \in \mathcal{V}$ and $q_{\ell}^{\prime}$ is unoccupied before the change. In both cases, $c_{\ell}(\cdot)$ reduces and $\mathrm{C}_{\mathcal{L}}(\cdot)$ also reduces. It is well known that BR dynamics converges to a pure-strategy $\mathrm{NE}$ if the game possesses a potential function [10].

\section{Swap Operation AND Pairwise Nash Stability}

By Propositions 1 and 2, vertex occupancy vector $\left(q_{\ell}, \mathbf{q}_{-\ell}\right)$ is a NE if and only if each vertex in $\mathcal{V}$ has one agent. Thus the game $\mathcal{G}_{\mathcal{L}}$ has a large collection of pure-strategy NEs. The task ahead is to promote NEs that have lower total cost $\mathrm{C}_{\mathcal{L}}\left(q_{\ell}, \mathbf{q}_{-\ell}\right)$, which translates to lower number of collisions among vertices of graph $G$. To this end, the paper develops a swapping mechanism that employes a stronger form of equilibria, called pairwise Nash stability [11].

Suppose the agents are in some initial pure-strategy NE occupancy state in $\mathcal{V}_{0}$, i.e., $\left|S_{v}\right|=1, \forall v \in \mathcal{V}$. Without loss of generality, let $\ell, \ell^{\prime} \in \mathcal{L}$ be occupying two distinct vertices in $\mathcal{V}, q_{\ell} \neq q_{\ell^{\prime}}$. Let $\mathbf{q}_{-\left\{\ell, \ell^{\prime}\right\}}:=\times_{\ell^{\prime \prime} \neq \ell, \ell^{\prime}} q_{\ell^{\prime \prime}}$, i.e., the strategies of all players except $\ell$ and $\ell^{\prime}$. Then consider the change in costs of the two agents $\ell, \ell^{\prime}$, and the change in total cost of the system, as $\ell$ and $\ell^{\prime}$ perform a swap (mutual exchange) of the vertices that they occupy, while all other players hold their initial vertices, i.e., $\mathbf{q}_{-\left\{\ell, \ell^{\prime}\right\}}$ remains constant. Let $q_{\ell}^{-}$(resp. ,$q_{\ell}^{+}$) denote the vertex occupied by $\ell \in \mathcal{L}$ before the swap operation (resp. after the swap operation). Note that $q_{\ell_{\sigma}}^{+}=q_{\ell^{\prime}}^{-}$, and vice-versa. The swap operation is denoted by $\ell \ell^{\prime}$. The change in cost of player $\ell$ is,

$$
\Delta c_{\ell}\left(\stackrel{\circlearrowleft}{\ell \ell^{\prime}}, \mathbf{q}_{-\left\{\ell, \ell^{\prime}\right\}}\right)=
$$

$a\left|\left\{v^{\prime}: t_{\ell} \in S_{v^{\prime}}, v^{\prime} \in \mathcal{V}_{q_{\ell}^{+}}\right\}\right|-a\left|\left\{v^{\prime}: t_{\ell} \in S_{v^{\prime}}, v^{\prime} \in \mathcal{V}_{q_{\ell}^{-}}\right\}\right|$.

The change in total cost is,

$$
\begin{aligned}
& \Delta \mathrm{C}_{\mathcal{L}}\left(\stackrel{\circlearrowleft}{\ell \ell^{\prime}}, \mathbf{q}_{-\left\{\ell, \ell^{\prime}\right\}}\right)= \\
& 2 \Delta \mathrm{c}_{\ell}\left(\stackrel{\circlearrowleft}{\ell \ell^{\prime}}, \mathbf{q}_{-\left\{\ell, \ell^{\prime}\right\}}\right)+2 \Delta \mathrm{c}_{\ell^{\prime}}\left(\stackrel{\circlearrowleft}{\ell \ell^{\prime}}, \mathbf{q}_{-\left\{\ell, \ell^{\prime}\right\}}\right) .
\end{aligned}
$$

This paper defines pairwise Nash stability of game $\mathcal{G}_{\mathcal{L}}$, following the pairwise Nash definition for network games in [11].

Definition 1. Strategy vector $\left(q_{\ell}, \mathbf{q}_{-\ell}\right)$ is pairwise Nash stable if it is a NE and if $\Delta \mathrm{c}_{\ell}\left(\ell \ell^{\prime}, \mathbf{q}_{-\left\{\ell, \ell^{\prime}\right\}}\right)<0$ then $\Delta \mathrm{c}_{\ell^{\prime}}\left(\stackrel{\circlearrowleft}{\ell \ell^{\prime}}, \mathbf{q}_{-\left\{\ell, \ell^{\prime}\right\}}\right)>0, \forall \ell, \ell^{\prime} \in \mathcal{L}$.

Definition 1 stays that the agents are pairwise Nash stable if they are in a NE and no pair of agents can swap their vertices to decrease both their costs. Thus two agents agree for a swap only if both obtain a reduction in costs. Thus we define the swap operation between agents as follows. 
Definition 2. Swap is the process where two agents mutually exchange vertices, if both agents obtain a strict reduction in costs.

Note that the occupancy state that results after each swap operation is a NE by Propositions 1 and 2. Mutual cost reduction is a stronger statement than BR. Swap operation can be employed to propel the system to a $\mathrm{NE}$ with better performance with regards to the social cost $\mathrm{C}_{\mathcal{L}}\left(q_{\ell}, \mathbf{q}_{-\ell}\right)$. It is important to theorize that swap may never increase the social cost and that the swap operation may not get stuck in an infinite loop, i.e., that sequential swaps reach a pairwise Nash stable coloring state after a finite number of steps.

Lemma 1. Swap always reduces social cost and only finitely many swap operations may happen before reaching pairwise Nash stability.

Proof: Note that the minimum reduction in cost by a change of action by a player is $a$. By Definition 2 and from (3), (4), if a swap operation takes place between two agents $\ell, \ell^{\prime}$, then $\Delta \mathrm{c}_{\ell}\left(\ell \ell^{\prime}, \mathbf{q}_{-\left\{\ell, \ell^{\prime}\right\}}\right) \leq-a$ and $\Delta \mathrm{c}_{\ell^{\prime}}\left(\ell \ell^{\prime}, \mathbf{q}_{-\left\{\ell, \ell^{\prime}\right\}}\right) \leq$ $-a$, which implies that $\Delta \mathrm{C}_{\mathcal{L}}\left(\ell \ell^{\prime}, \mathbf{q}_{-\left\{\ell, \ell^{\prime}\right\}}\right) \leq-4 a$. Thus, every swap operation reduces the system cost by at least $4 a$. However the system cost $\mathrm{C}_{\mathcal{L}}(\cdot)$ is lower bounded by 0 . Therefore only a finite number of swaps are possible. Once all pairs of agents have consecutively failed a successful swap, by Definition 1 the players have reached pairwise Nash stability and swap process terminates.

By Lemma 1, sequential swap operation always converges to a local minima of system $\operatorname{cost} \mathrm{C}_{\mathcal{L}}(\cdot)$. In a HetSNet with a large number of vertices, sequential swap operation may not be ideal. However as discussed next, swap operation can be parallelized with certain restrictions that preserve Definition 2.

Let us consider a NE state where agents $\ell, \ell^{\prime} \in \mathcal{L}$ occupy two distinct vertices in $\mathcal{V}$. The cost of an agent $\ell$ is a function of only the adjacent agents denoted by $\mathcal{L}_{\ell}:=$ $\left\{\ell^{\prime \prime}:\left(q_{\ell}, q_{\ell^{\prime \prime}}\right) \in \mathcal{E}\right\}$. Therefore, if $\ell, \ell^{\prime}$ decides to swap, the agents occupying the immediate neighbor vertices of $q_{\ell}$ and $q_{\ell^{\prime}}$ must remain static. Otherwise the costs of the two agents $\ell, \ell^{\prime}$ cannot be guaranteed to reduce thus possibly violating Definition 2 . Therefore the agents occupying neighbor vertices of the $\ell, \ell^{\prime}$, are in a restricted state. However two other agents who occupy vertices that do not share an edge with the vertices $q_{\ell}$ and $q_{\ell^{\prime}}$, may engage in their own swap and in turn the restricted state applies to their neighbors. Parallel swap operation is scalable for a large network, as the number of nodes grows, but as long as the edges remain sparse. In the worst case, if the network is a click, i.e., every node is connected to all other nodes, only sequential swap is possible.

\section{REFINING EQUILIBRIA BY REFEREEING}

One drawback of the swap operation is that the agents who are initially occupying the virtual vertex $v_{0}$, do not get a chance. Because no agent occupying a vertex in $\mathcal{V}$ can reduce its cost by swapping with an agent in $v_{0}$. Therefore the final occupancy state of the colored agents depends on the initial NE occupancy state from which the swap operation began. To allow the agents in $v_{0}$ to explore graph $G$, the involvement of a referee is required. A referee is a process that intervenes externally to modify the outcome [12]. The task of the referee is to forcefully swap agents occupying vertices in $\mathcal{V}$ with agents occupying the virtual vertex $v_{0}$. The refereeing process in itself can be modeled as a complex combinatorial optimization problem with knowledge of the percent occupancy state of the graph $G$. However for the sake of this paper we propose the following simplified refereeing process.

Definition 3. Referee picks an agent $\ell$ occupying a vertex $v \in \mathcal{V}$ and replace $\ell$ with an agent $\ell^{\prime}$ occupying $v_{0}, \quad$ if $\quad \mathrm{c}_{\ell^{\prime}}\left(q_{\ell}=v_{0}, q_{\ell^{\prime}}=v, \mathbf{q}_{-\left\{\ell, \ell^{\prime}\right\}}\right) \quad<$ $\mathrm{c}_{\ell}\left(q_{\ell}=v, q_{\ell^{\prime}}=v_{0}, \mathbf{q}_{-\left\{\ell, \ell^{\prime}\right\}}\right)$.

Note that, unlike swap, referee violates the mutual cost lowering requirement of Definition 2. Therefore after refereeing, swap operation has to be carried out to bring the state back to a pairwise equilibrium. Lemma 2 establishes that the referee is innocuous and that it terminates. A referee is innocuous, if after the refereeing operation, the social cost of the graph cannot increase.

Lemma 2. Occupancy state after the refereeing operation is a $N E$, has system cost $\mathrm{C}_{\mathcal{L}}(\cdot)$ no greater than the cost before refereeing, and halts in a finite number of steps.

Proof: Proof that the state after refereeing is a NE follows from Propositions 1 and 2. To notice that the system cost cannot increase, consider that $\ell$ is the agent that is forced out to $v_{0}$ and $\ell^{\prime}$ takes the previous vertex of $\ell$. Since referee satisfies Definition 3, the number of neighbor in collision with $\ell^{\prime}$ is less than or equal to the number of collisions $\ell$ had. The halting of the refereeing process follows by a similar argument to that of halting of swap operation in Lemma 1.

Swapping coupled with the refereeing operation provides a powerful combination that can escape a local minima, which has a higher social cost, and explore further in the space of all possible coloring states for pairwise stable colorings with lower social cost. If need be it is possible to define the referee such that it creates additional agents, or change the color of an agent, when it cannot find agents in $v_{0}$ that satisfy the requirements of Definition 3. The referee function can be implemented fully distributively by a software robot which visits BSs in random or centrally by a leader of the colored agents, that is elected at an initialization stage.

\section{NUMERICAL RESUlTS}

We explore the results through a frequency band assignment problem. To minimize cochannel interference, similar frequency bands should not be allocated to adjacent BSs. The interference relations between BSs are generated randomly. Both $a$ and $b$, of cost function, are set to 1. Fig. 2 validates Proposition 3. The network has 20 BSs. Initially the frequency agents are assigned to the BSs randomly, therefore it is probable that there are BSs with multiple agents and/or no agents. Then a sequential process selects agents to perform BR dynamics. This simulation considers two selection processes, 


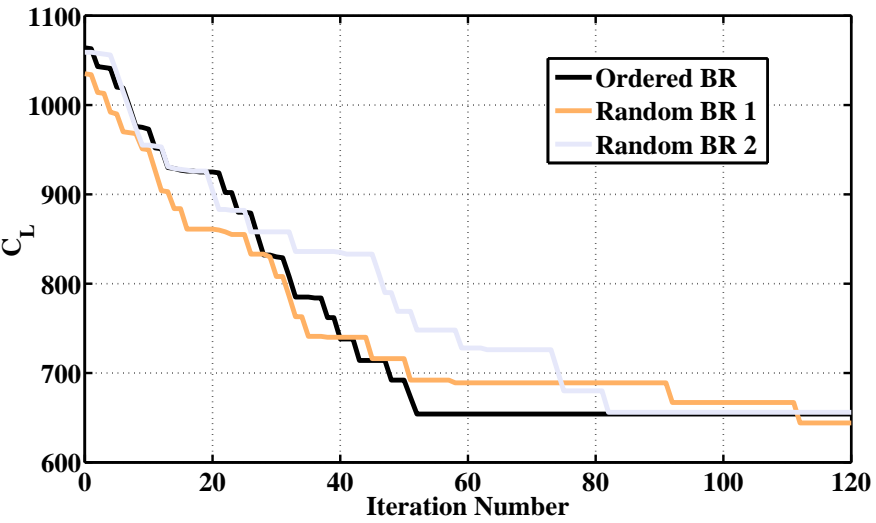

Figure 2. Convergence of BR dynamics depends on the initial starting point and agent selection process.

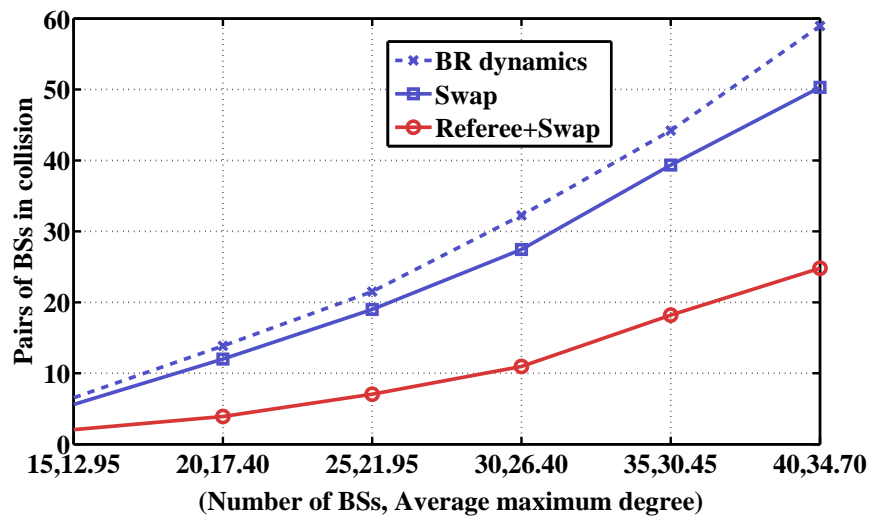

Figure 3. Performance improvement through swap and referee operations.

sequential by index and random. The process terminates when none of the agents are able to perform a BR.

Fig. 3 demonstrates the reduction of cochannel collisions, by swap and referee operations. Only 10 frequency bands are available and each frequency is duplicated 10 times, creating 100 frequency-agents. The simulation starts-off from a random one-to-one allocation of frequency-agents to $\mathcal{V}$ and the rest of the agents occupying $v_{0}$. Then swapping takes the network to pairwise Nash stability. Finally refereeing operation is carried out together with swapping. The $\mathrm{x}$-axis shows the number of BSs and the maximum degree, $\Delta(G)$ of the interference relations. As is seen from the figure, the referee and swap operations together achieve phenomenal performance, given that the test networks have high $\Delta(G)$.

Fig. 4 demonstrates the effect of the number of frequency bands in reducing the collisions. Number of BSs in $G$ is 40 . The total number of agents is kept constant at 100. As the number of frequency bands increases all three schemes show a reduction in the collisions, which is expected.

\section{CONCLUSION}

This paper considers the problem of allocating a finite resource, such as frequency bands and time slots, over a set of BSs, in order to minimize the pairs of adjacent BSs that are in collision. The paper follows a game theoretic approach.

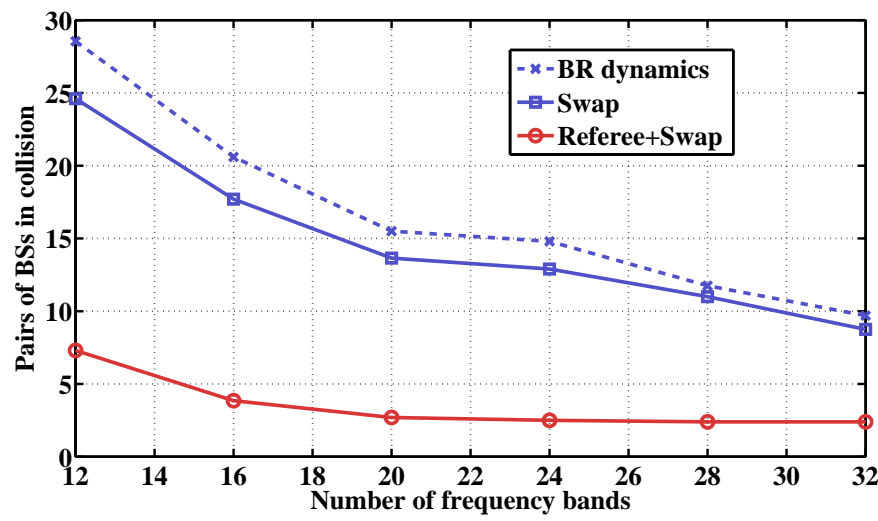

Figure 4. As the number of colors increases the performance of all schemes improves.

The game is played among a set of agents, who represent the resources. It is demonstrated that the game possesses a multitude of pure-strategy Nash equilibria. Therefore two refining mechanisms are developed. First refining process, swaps pairs of agents and in the end achieves a pairwise Nash stable coloring. Further improvement is achieved by an external referee, wh0 helps the resource agents to explore assignments that would not have been explored in swapping. The simulation results demonstrate that refereeing coupled with swapping vastly out performs best response dynamics in average. Practical implementation of agents by software robots in a test bed and weighted case of this problem, where the BSs have priorities, need to be explored in future work.

\section{REFERENCES}

[1] I. Hwang, B. Song, and S. Soliman, "A holistic view on hyper-dense heterogeneous and small cell networks," IEEE Commun. Mag., vol. 51, no. 6, pp. 20-27, 2013.

[2] M. Peng, D. Liang, Y. Wei, J. Li, and H.-H. Chen, "Self-configuration and self-optimization in LTE-advanced heterogeneous networks," IEEE Commun. Mag., vol. 51, no. 5, pp. 36-45, May 2013.

[3] P. Formanowicz and T. K, "A survey of graph coloring - its types, methods and applications," Foundations of Computing and Decision Sciences, vol. 37, pp. 149-238, Oct. 2012.

[4] J. Riihijarvi, M. Petrova, and P. Mahonen, "Frequency allocation for WLANs using graph colouring techniques," in Wireless On-demand Network Systems and Services, 2005. WONS 2005. Second Annual Conference on, Jan 2005, pp. 216-222.

[5] P. N. Panagopoulou and P. G. Spirakis, "A game theoretic approach for efficient graph coloring," in Proc. 19th International Symposium on Algorithms and Computation, (ISAAC '08), 2008, pp. 183-195.

[6] I. Chatzigiannakis, C. Koninis, P. Panagopoulou, and P. Spirakis, "Distributed game-theoretic vertex coloring," in Principles of Distributed Systems, ser. Lecture Notes in Computer Science. Springer Berlin Heidelberg, 2010, vol. 6490, pp. 103-118.

[7] B. Escoffier, L. Gourves, and J. Monnot, "Strategic coloring of a graph," Internet Mathematics, vol. 8, no. 4, pp. 424-455, 2012.

[8] K. Chaudhuri, G. Chung, and M. Jamall, "A network coloring game," in Proc. Internet and Network Economics, ser. Lecture Notes in Computer Science. Springer Berlin Heidelberg, 2008, vol. 5385, pp. 522-530.

[9] M. Halldorsson, J. Halpern, E. Li, and V. Mirrokni, "On spectrum sharing games," Distributed Computing, vol. 22, no. 4, pp. 235-248, 2010.

[10] N. Nisan, T. Roughgarden, E. Tardos, and V. V. Vazirani, Algorithmic game theory. Cambridge University Press, 2007.

[11] M. Jackson, Social and Economic Networks. Princeton University Press, 2010.

[12] Z. Han, Z. Ji, and K. Liu, "Non-cooperative resource competition game by virtual referee in multi-cell ofdma networks," IEEE J. Sel. Areas Commun., vol. 25, no. 6, pp. 1079-1090, Aug. 2007. 